\title{
Ontological Model of Multi-Source Smart Space Content for Use in Cultural Heritage Trip Planning
}

\author{
Kirill Kulakov, Oksana Petrina \\ Petrozavodsk State University \\ Petrozavodsk, Russia \\ kulakov@petrsu.ru, petrina@cs.karelia.ru
}

\begin{abstract}
This paper presents ontological model for storage of cultural heritage and trip planning information in Smart Space. This model combines external information service-specific ontologies to the one generic ontology and provides easy way to construct distributed Smart Space-based service from various modules. Cultural heritage information presented in ontological model is used to search and recommend attractions. The paper contains proposed high-level service architecture and description of various use cases which are verified presented ontological model.
\end{abstract}

\section{INTRODUCTION}

Tourism is one of the popular areas for software development. Tourists can use various applications for gathering required information before the trip (trip planning) or during the trip (trip support). One way of obtaining information is to use recommender system. There are a lot of recommender systems for various cases [1].

The main problem of most recommender systems is using actual information. A lot of data is dynamically updated during the trip and the tourist should know about these changes and plan the changes in the trip. Another problem is to find attractions of interest. Commonly the tourist knows some attractions in the target region and he/she wants to search other attractions from different sources. In this case the recommender system is preferred, but it requires a lot of information about the tourist (preferences, list of visited attractions, etc.).

One way to solve this problem is to use Smart Space technology for service development. It provides possibility to create common information space, proactive concept and flexible connections to external sources [2]. The one way to implement Smart Space-based service is to use Smart-M3 platform [3]. The Smart-M3 platform is based on semantic information broker (SIB), which collects and distributes information, and knowledge processor (KP), which represent external information sources or consumers and perform operations on the knowledge. The one of the steps of implementation is to create common information space based on ontological model.

In the paper [4] an approach to creation of Smart Spacebased trip planning service is described. This approach includes high-level architecture, trip planning problem description, high-level architecture and usage scenarios descriptions. This service includes recommendation function to search and recommend attractions. An interesting case is recommendation for such a particular tourism domain as historical and cultural tourism. The one of such recommendation approaches is described in the paper [5]. This approach includes mathematical model, cultural information description and highlevel architecture with required modules. One of the steps in implementing these approaches is to create a common information space. It requires data model based on ontological principles with descriptions of its usage.

In this paper we provide ontological model for storage of cultural heritage and trip planning information in smart space. The model combines external information service specific ontologies from trip planning and cultural heritage recommendation areas and presents generic ontology. The presented ontological model describes single tourist personal space.

The trip schedule presented as a set of movement between points. This approach allows to easy include new activities or recommended attractions. Ontological model also includes classes to describe cultural heritage, climate time and movement restrictions. These classes can be supplemented by new properties or other restrictions.

The model uses to create common information space for cultural trip planning service, which is based on approaches from our previous papers [4], [5]. In the trip planning service each external information service presents as a module with KP. Intermodule communication with generic ontology is presented by various use cases: creating trip schedule using service core, gathering attractions and events and additional sources usage. These use cases describes basic scenarios for using proposed trip planning service.

The rest of the paper is organized as follows. Section II presents related work. Section III describes proposed common service architecture. Section IV presents the list of use cases. Section V describes ontological model structure. Section VI summarizes contribution of this paper.

\section{RELATED WORK}

Paper [6] presents an ontology based approach for developing STAAR (Semantic Tourist information Access and Recommending). STAAR help tourists to search the information by providing a various semantic search features in a mobile phone application. Ontology is used to simulate all the data and knowledge stored in the system STAAR, including tourist's profile, description of tourist resources and services. Ontology defines primitive concepts, properties for representing tourism, related semantic annotations, formalized by using RDF/RDFS language. Own system data is imported with relevant external data from external sources DBpedia, GeoNames in the form of triples. But this ontology cans not be used to create a schedule. 
Our service involves the creation of a cultural program for tourists.

Paper [7] presents a multi-stage ontology based touristic recommender and information retrieval system for Interactive Community Displays. The system is able to offer customized advice for citizens and tourists, including those with special needs. Different modules of the system are combined into a total of Ontology. A user feature might be any perceivable attribute or behaviour of the user, observed within the user profile ontology. The user profile is stored according to the profile ontology. Also the ontology takes into account the context as class Context which comprises subclasses Day, Address, CurrentTime, Temperature, Meteorology. The ontology has classes which take into account the traffic situation e.g. TransportType, TransportAccess, ActivityCost. The paper presents an example of an ontology for the case of a restaurant. This system not implies the extract of historical information from external sources resp. the ontology is also not designed for this.

Paper [8] describe the architecture, the knowledge model that is shared among components using an ontology of the user-oriented adaptive system SAMAP for planning tourist visits. High level view of the ontology developed for SAMAP consist classes User, Personal preferences, City, Transport, Activity, Visit, Place. Class User has attributes about the personal information of the user like name, profession, mobility, language, and etc. Class City is represented together with classes that describe transport means, places that can be visited, as well as the streets that compose the map of a city. Class Place defines specific sites within a city that can be visited, such as restaurants, museums, generic buildings, bars, openspaces, or theatres. This ontology is a good description of the necessary aspects for tourists but it ignores type of trip and type of road.

Paper [9] presents SigTur/E-Destination, a tourism recommender system. Ontology in the Tourism domain is structured around eight main concepts Events, Nature, Culture, Leisure, Sports, Towns, Routes and ViewPoints. This ontology has been developed using the Thesaurus of the World Tourism Organization. The ontology only contains classes that permit to describe types of activities. This ontology is suitable to provide users recommendation, but does not support schedule.

Paper [10] presents approach to coating the touristic semantic space. The coating is carried by the integration of modular ontologies, for example, W3C Time and W3C Geo. W3C Time is a vocabulary for expressing facts about topological relations between moments and intervals, along with information about the duration, as well as the date and time information. W3C Geo is the main RDF vocabulary for the description of latitude and longitude. We use W3C Geo to development our ontology.

Paper [11] describes a partial ontological model for cultural spaces and the approach to link that model to services useful for devising touristic offerings. This ontology is a good description points of interest (POIs) and historical information for them, but is not describes aspects related to trip planning.

In the last decade, numerous attempts to create historical ontologies have been made, see [12], [13], [14]. For instance, ontology CIDOC CRM [15] is specially designed for the description of museum artifacts. It can be effectively employed to arrange knowledge about historical persons and events. Despite obvious needs, specialized historical knowledge bases are not available on the Semantic Web up to now.

\section{SERVICE ARCHITECTURE}

The high-level architecture of cultural trip planning service is based on [4], [5] and presented in Fig. 1. The service is based on Smart-M3 platform distributed architecture and each unit commonly works on separate device. The KP provides access to external information service and translates obtained information to the common space. The SIB is a semantic information sharing service, providing semantic level communication channel for KPs to interact with each other. Our architecture design decisions also use the experience of papers [16], [17], [18].

User KP is responsible for providing information about the user and users preferences and gets results trip planning service. User KP is combines the functions of User KP from [4] and Client KP of [5] and represents user's positions, personal data and information about user's preferences, and core attractions and planning restrictions. User KP publishes personal data and information about user's preferences and subscribes to recommendations related to POIs. It publishes the fact that the user has attended a given POI and also publishes an evaluation of the POI. This feedback can be used by the application to tune ranging algorithms. Also User KP provides result of route planning algorithm to the tourist.

We aim to use five data source KPs: WeatherKP, GeoInformationKP, EventKP, GeoPositionKP, and BookingKP. These KPs get information from public data sources and publish data (or references to the data) in the smart space (via the SIB) for shared use by other KPs. The name "data source KPs" was taken from [5] but the components are significantly different. Most of these KPs have been described in [4] however GeoInformation KP is supplemented by special functions.

GeoInformation KP provides information about attractions (position, description, images, etc.) from third-party services. Also route planning algorithm requires estimations for attractions (e.g. schedule, inspection time or attraction size). This information can be estimated or provided from other tourists. GeoInformation KP function controls have been advanced features for working with knowledge bases. More details can be read in [5]. Now also GeoInformation KP provides the smart space with cultural heritage data. This KP is oriented to ontology of a definite knowledge base (Dbpedia, Freebase). It is provided with a certain set of query templates that can be put to a knowledge base. The application for mobile cultural tourism is designed to process data extracted from knowledge bases.

Weather KP provides current and future weather for requested coordinates from third-party weather service. Event KP provides information about events (position, description, schedule, etc.) from third-party services. Event KP should detect region for required position and provide up-to-date events to SIB, as the route planning algorithm should not consider past events. Booking KP provides information about hotels and other accommodations (name, place, photos, price, availability, etc.) from third-party services. Transport KP provides information about transport: schedule for public transport 


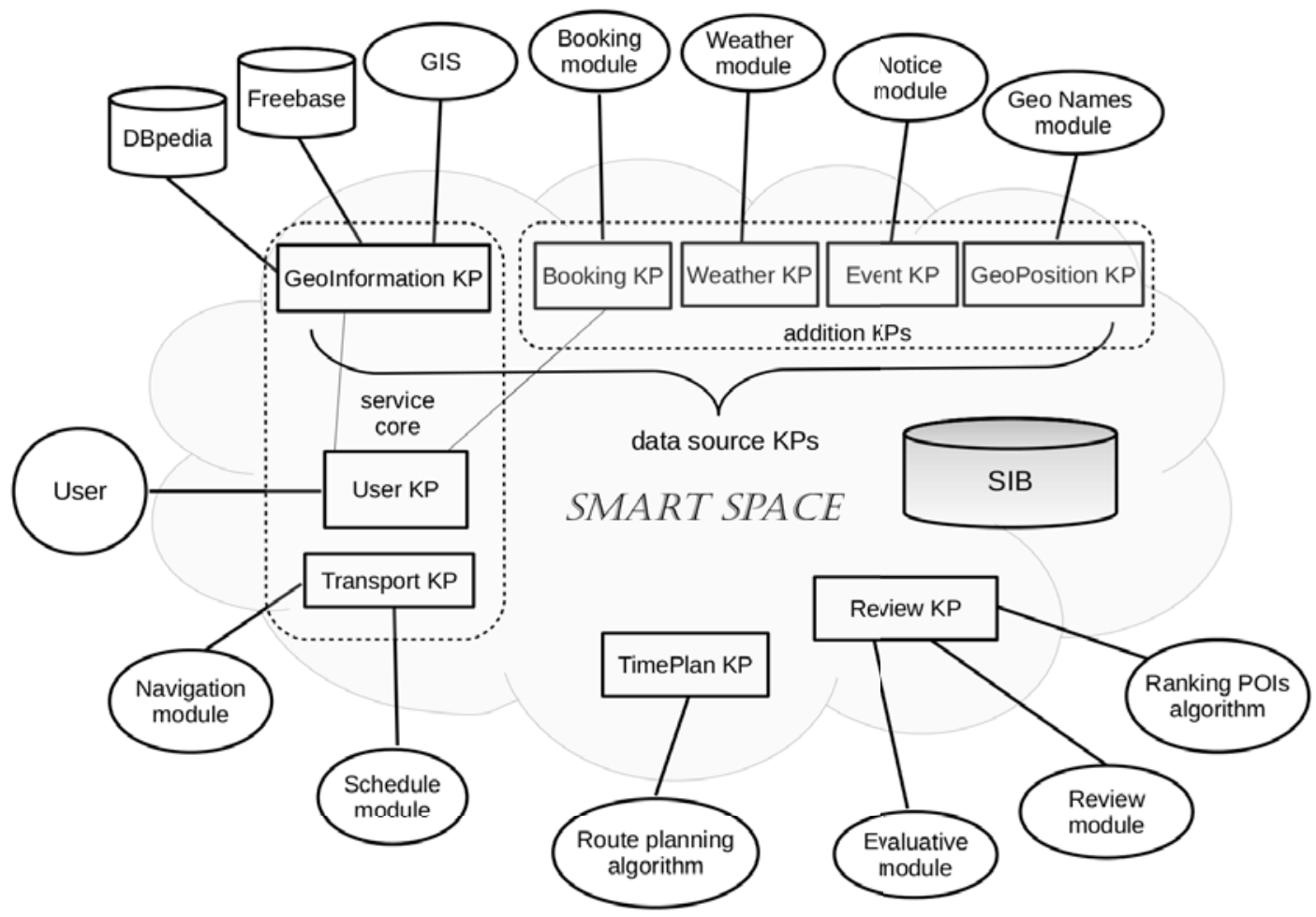

Fig. 1. Cultural trip planning service high-level architecture

(e.g. bus, ship, train), routes from third-party services, internal road map and other conditions for selected transport (drive time, stops, etc.). The Transport KP provides optimal route for required set of points and options for each part (time, speed, distance).

SIB aggregates information about POIs. It constructs a semantic information structure that combines data retrieved from different Data Source KPs for each POI.

Review KP is combines the functions of Review KP from [4] and Ranking KP of [5]. The Review KP provides required estimations, which can be evaluated using internal Evaluative service or obtained as the average value from thirdparty Review service. The Review KP compoutes ranks of POIs on the basis of combined information about the POIs. The $\mathrm{KP}$ subscribes to the users preferences and the visiting history, and also evaluates duration of the POI visit. Then it publishes the ranked list of POIs drawn up for the user. Each candidate POI becomes assigned to the rank value. Then the User KP can use these values for representation of recommended POIs to the user. It should be noted that the methods for information ranking and evaluation of users preferences vary considerably. Therefore Review KPs can employ different algorithms as well as their combinations.

TimePlan KP is a mediator and implements Route planning algorithm. It uses information from other KPs and provides route plan to User $K P$.

This architecture can be changed by adding or removing KPs and modules. The minimal configuration (service core) includes User KP, GeoInformation KP, Transport KP with Navigation service and mediator TimePlan KP.

\section{USE CASES}

According to our approach [4] we describe the following scenarios using ontology model.

1) Creating trip schedule using service core. Tourist defines target points and smart space-based trip planning service creates schedule based on the route info.

2) Gathering attractions and events. Tourist prepares request parameters and performs a search query to external data sources.

3) Additional sources usage. In this case service also creates schedule, but it uses additional sources to improve its accuracy.

The first and second scenarios are basic and requires service core. The result of the last scenario depends on available KPs. If there are no additional KPs available, then the second and the third scenarios works equally.

Each scenario is focused on the use before trip (trip planning) and during the trip (trip support). It is assumed that before trip user has good access to the Internet and can perform large number of search queries, but during the trip user has unstable access to the Internet and use service to obtain schedule updates. Therefore, most of used information (like attraction descriptions and images) are downloaded to tourist mobile device before trip and updated during the trip.

This scenarios use two procedures:

- "publish/remove" — the KP publishes required information or removes unused information in the SIB, if it's required; 
- "subscribe" - the KP subscribes to required information and receives it after changes in the SIB.

According to the Smart Space technology, the result of query can be iteratively updated with the appearance of additional information. This causes that user doesn't obtain good result immediately, the service notifies User KP about result updates. Therefore any KP can publish updated data to the SIB and start new thread of scenario from corresponding step.

Each scenario demonstrates by corresponding sequence diagram. The diagram shows data flows between KPs and SIB. The parentheses describes links between class individes which are important in this data flow: $\mathrm{A}(\mathrm{B})$ means that class A has link to class $B$. The square brackets with asterisk presents a set of class individes and this set may be empty: $\mathrm{A}\left(\left[\mathrm{B}^{*}\right]\right)$ means that individe of class $\mathrm{A}$ has a set of links to individes of class B.

\section{A. Scenario 1: Schedule preparation using service core}

The sequence diagram of scenario 1 is presented in Fig. 2 . This scenario starts from creating the list of required attractions

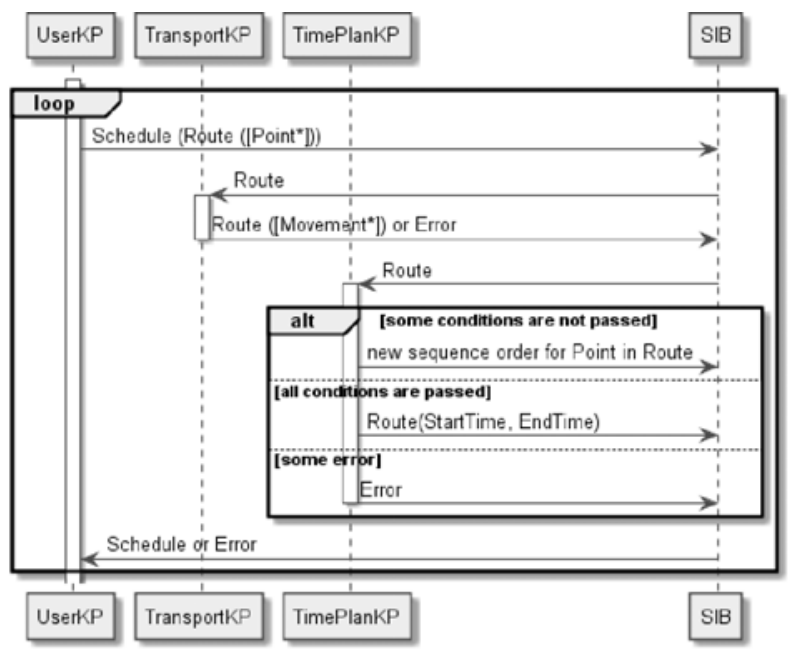

Fig. 2. Schedule preparation using service core

and events. The UserKP publishes the Schedule individe, which includes Route individe with set of Point individes.

The TransportKP subscribes to the Route class and creates route as a list of movements which includes start point, end point and route type. Accurate route does not need to be stored in the SIB, it can be displayed to the user by UserKP independently. These movements are published to the SIB as a set of Movement individes in Route individe. If some point does not achievable, then TransportKP publishes Error individe.

The TimePlanKP subscribes to the pair Route-Movement and publishes timetable as a pair StartTime and EndTime in Movement class. If time conditions was mismatch then TimePlanKP prepares new sequence of points and publishes it to the SIB. The TransportKP prepares route for the new sequence and TimePlanKP checks conditions again. This process works iteratively until correct sequence is found or proved the absence of solution. The UserKP collects timetable for requested Schedule or error if it caused and displays it for user.

This process is iterative. For example, user can modify list of required attractions and events.

\section{B. Scenario 2: Gathering attractions and events}

The sequence diagram of scenario 1 is presented in Fig. 3 . This scenario starts from publishing of personal information by

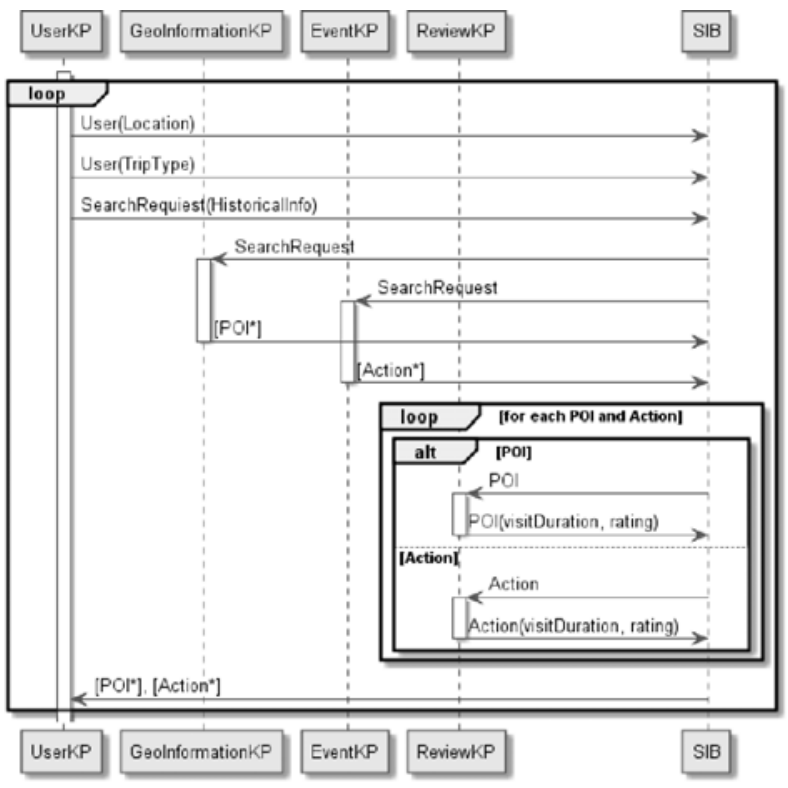

Fig. 3. Gathering attractions and events

UserKP. This information includes the user's location (individe of Location, detected by mobile device), trip type (individe of TripType, selected by the user) and search request (individe of SearchRequest). The request can include region restrictions, name and other cultural filters corresponding ontology.

The GeoInformationKP and EventKP subscribe to the SearchRequest class and start searching process after publishing of individes by each KP. After finishing of search process the result is published to the SIB (individes of classes POI and Action). The UserKP subscribes to updates SearchRequest individe updates and presents found attractions and events to the user.

This process is iterative: the user can clarify search request.

\section{Scenario 3: Additional sources usage}

This scenario extends Scenario 2 by using additional thirdparty sources and restrictions. The scenario is divided into the following cases.

1) "All conditions was passed" (see Fig. 4). In this case GeoPositionKP subscribes to the Point class and publish its address for each point. The WeatherKP subscribes to the points with address and returns weather forecast for selected time duration. In parallel, TransportKP prepares route for required points 


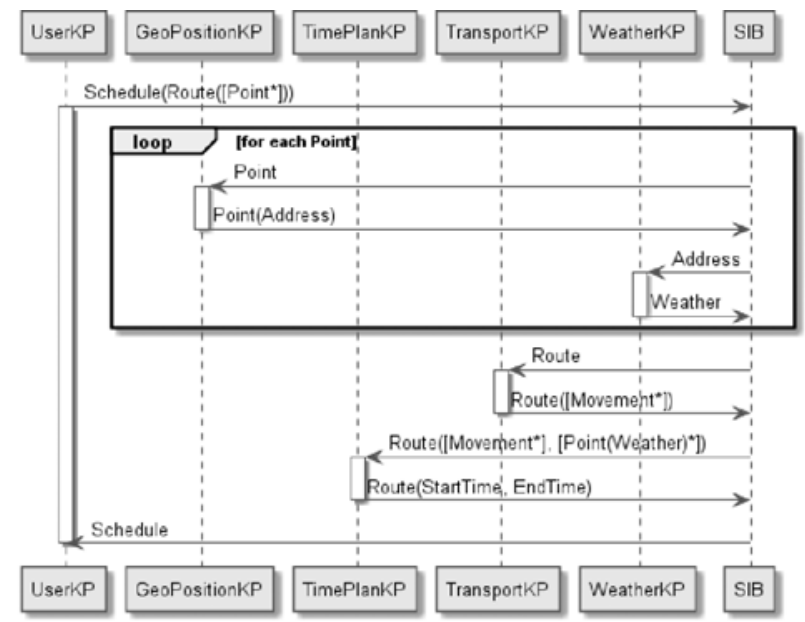

Fig. 4. Using weather conditions

according to Scenario 2. At last, TimePlanKP checks time and weather conditions and creates timetable.

This process is also iterative because weather forecast may change and WeatherKP should request data iteratively.

2) "Movement condition are not passed" (see Fig. 5). This case starts similar to the first, but TimePlanKP

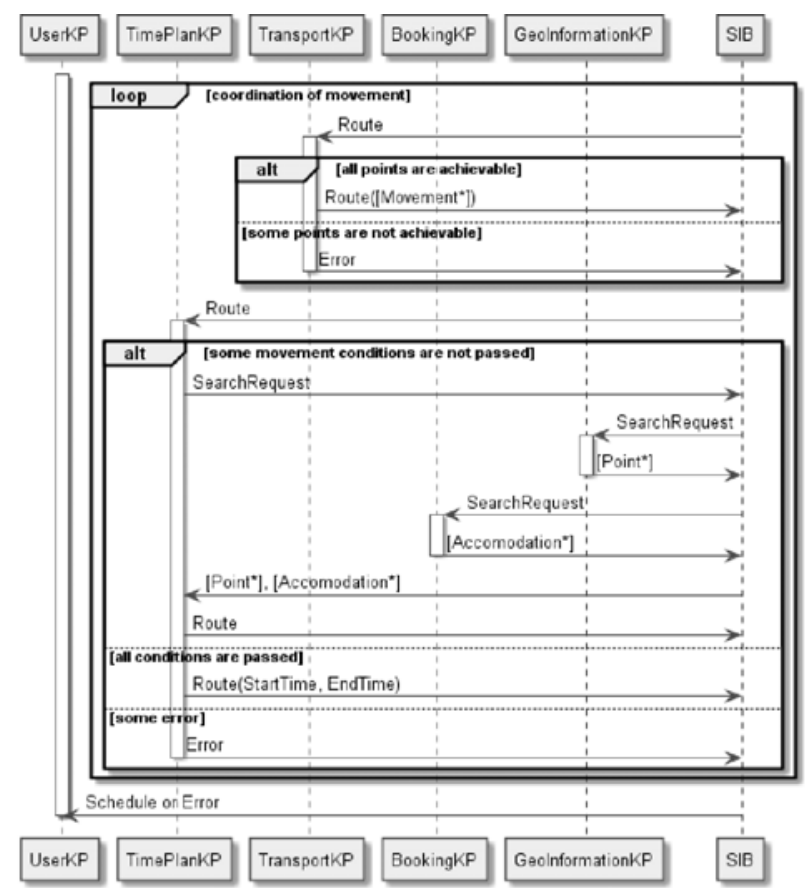

Fig. 5. Coordination of movement

finds mismatch movement conditions. In this case, TimePlanKP prepares additional SearchRequest to found points near incorrect movement. The GeoInformationKP and BookingKP subscribe to the SearchRequest class and publish found accommodations, pit stops and attractions. Then TimePlanKP selects some founded points and add it to the Route class. This pro- cess works iteratively until all movement conditions are passed or error is caused.

\section{ONTOLOGICAL MODEL}

We present an ontology based on the architecture and use cases. The ontology unite the tourist information: POIs description and tourist events, visit schedule, weather data and transport, accommodation and other useful information. Historical information collects for POIs. It gets from universal knowledge bases, such as Dbpedia and Freebase.

Ontology encompasses several important aspects in the field of tourism, including tourist places (cities, towns), tourist attractions, tourist events (concerts, shows, etc.), as well as transport issues. Classes and subclasses of the ontology are shown on Fig. 6.

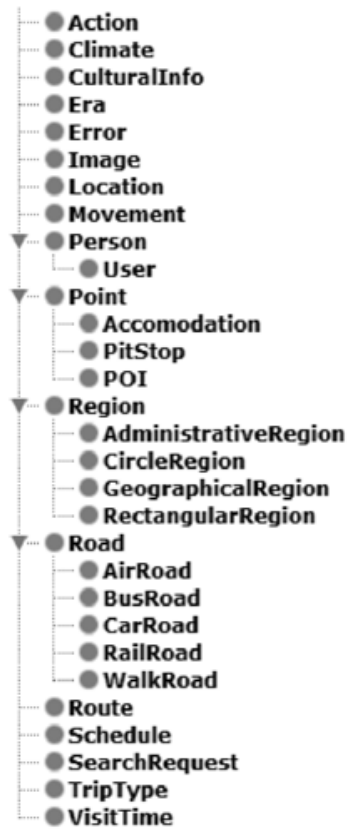

Fig. 6. Classes of the ontology

The best route to travel provides user as a result of the service. All the features of the service are displayed in the ontology. Appearance ontology visualized presented at on Fig. 7.

The user receives a schedule. Class Schedule linked to class Route with object property hasRoute. Class Route is route points (hasPoint) and movement (hasMovement).

Class Movement is one moving between two points. Movement has starting point and ending point. So class Movement linked to class Point with two object properties isStartPoint and isEndPoint. Classes Movement also has the designation starting and ending time with two data properties startime and endTime.

Class User is linked to class Point with two object properties hasVisitPoint and hasFavoritePoint. Thus, the user may mark POI that he visited and that he wants to visit. Class User 


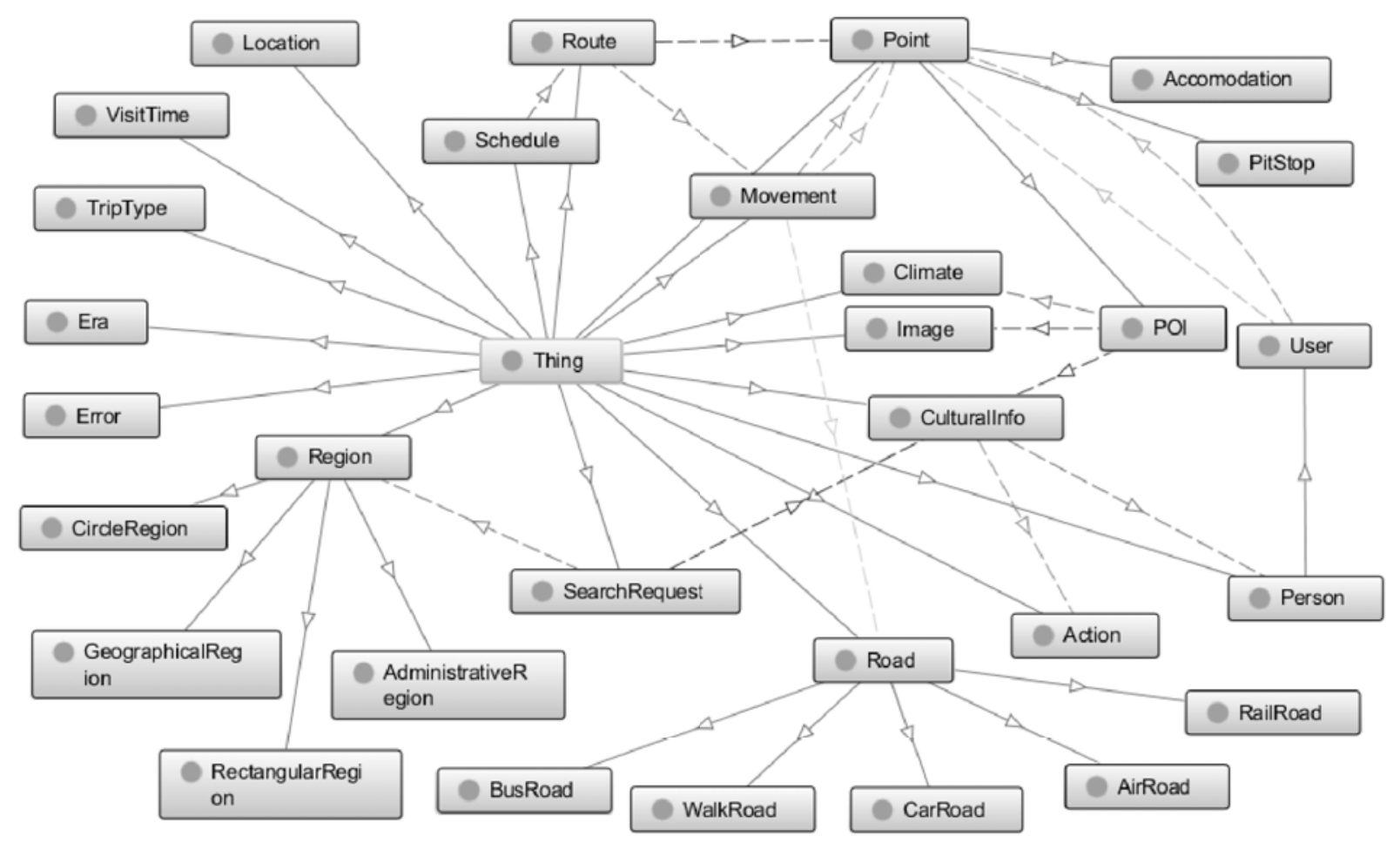

Fig. 7. Visualization of ontology

has two data properties login and password needed for user authentication in the system.

Class Point is a point of the route, having four subclasses. Subclass of Point Accomodation such as Hotel, Campsite, Hostel, Cottage, etc. Subclass of Point PitStop is technical stops during the travel.

Class $P O I$ is a point of interest. This class has data properties POICategory, rating, visitDuration. POIs Categories are building, monument, park, street, and et. The user may notes interesting for yourself POIs categories. Data property rating is need for user recommendations. The user receives personalized recommendations depending on the rating POIs.

Class POI linked to class Climate (hasClimateRestriction), to Image (hasImage), and to CulturalInfo (hasCulturalInfo). The weather is important for route planning. Some places demand for visiting certain weather conditions. Class Climate describes climatic characteristics. This class has three data properties meteoConditions, temperature, weatherSeason. Data property meteoConditions describes different weather conditions, such as sun, rain, snow, and etc. Temperature describes air temperature in degrees Celsius. WeatherSeason is convenient season for visiting the POI.

Class Image is different photos from external services such as Panoramio or Flickr and others. Thus, the user can view photos of POIs. Photos help the user to get a representation about the place which can be visited.

Class Culturalinfo is cultural heritage profile of the object of interest. This class is used for search queries to external data sources. This class has data property architecturalStyle which also used as user preference class User. Data property author may be an architect, a sculptor, and etc. for POI. Data property creationDate may be a date of emergence, date of Construction, and etc.

Class Action describes cultural events and activities. Any Action has starting time (startTime) and ending time (endTime). Class CulturalInfo is linked to class Action (hasAction) and Person (relatedWithPerson). Classes Action, POI, Image and CulturalInfo have data property description. Class Person contains a subclass User. The user may write his personal data. Based on these data class Person has data properties name, DateOfBirth, hometown, profession.

Class Location present location in the form of geographical coordinates and postal address. Geographical coordinates correspond WC3 Geo with data properties lat and long to designate geographical latitude and longitude, respectively. Class Location has one data property address which is a postal address location.

Class Era is an epoch in which belongs POI, for example, the Renaissance or the Postmodernism. Any concrete century or a significant event also can relate to Era, for example, XIX century or The French Revolution. There is required explicitly specify a concrete time frame. Therefore class Era has two data properties startTime and endTime.

Class Movement linked to class Road with object property useRoad. Class Road is type of road / transport. Depending on type may have different terms of travel. Having five subclasses: AirRoad, RailRoad, BusRoad, CarRoad, WalkRoad. Each subclass is different conditions of movement. AirRoad implies fast and nonstop way to travel. RailRoad implies less fast way to travel, the possibility of a comfortable sleep with fixed stops. 
BusRoad implies also way to travel with fixed stops but less comfortable than RailRoad. CarRoad implies the possibility to anystop, change of route, and other mobility. WalkRoad shoulds take into account the proximity of POIs and weather conditions. Now we do not specify details of the realization of these features but they may be added later.

Class Region having four subclasses. Subclass AdministrativeRegion may be country, region, state. SubclassCircleRegion is coverage area - the circle (excluding the spherical surface), has data property radius. Subclass GeographicalRegion may be as lake, forest, field. RectangularRegion is coverage area the circle (excluding the spherical surface) and has two data properties height and width.

Class TripType is type of travel, for example, eco-tourism, cultural tourism, gastronomic journey, etc. Type of travel take into consideration in the preparation of recommendations. For Example, tourist with a gastronomic journey is interested in restaurants and a local cuisine, but tourist with a cultural tourism is interested in cultural heritage and etc.

Class SearchRequest save template queries to service external data sources. For each source will be made the request based on user needs. Request reflects user preferences, location, type of travel. The result of the request is most interest POIs to the user.

Class Error is designed to handle errors, for example, when it is impossible to plan a route or there is no access to data source or there is no answer to a search query and so on. Class Error has three data properties errorCode, errorDescription, errorMessage.

The domain ontology has been developed with the Protégé editor and represented in the OWL language. The ontology contains 30 classes (concepts), 32 data properties and 19 object properties which indicate the relationships among the 30 classes.

\section{CONCLUSION}

Implementation of the cultural heritage trip planning service with supporting of many external sources is a complex problem. The knowledge processor requires various data which can be inferenced by other information or result of other knowledge processors work. Presented ontological model provides structures and relationships for trip planning and cultural heritage information storage in Smart Space.

Proposed model describes generic ontology and can be easy extended by adding new classes and relations. The common advantage to use generic ontology is that all knowledge processors (KPs) will understand required information, published from other KPs. The model does not constrain the possibility of KP, each KP can publish additional information for own using or for other KPs.

The ontological model can be used to construct distributed Smart Space-based service from various modules. The presented architecture combines cultural heritage and trip planning approaches and allows to use various external information services. All modules are divided into service core which is a minimal configuration and additional KPs. The modules from additional KPs extends information in Smart Space and allows to improve service results.
This model is verified by basic usage scenarios. These scenarios includes trip preparation and investigation of the target region (scenario 2), schedule constructing based on selected attractions with using minimum external services (scenario 1) and possibility of using additional external service to improve result. Proposed scenarios are flexible, most thirdparty services can be disconnected without significant changes during the process.

\section{ACKNOWLEDGMENT}

This research is financially supported by the Ministry of Education and Science of the Russian Federation: project \# 14.574.21.0060 (RFMEFI57414X0060) of Federal Target Program "Research and development on priority directions of scientific-technological complex of Russia for 2014-2020" and project \# 2.2336.2014/K from the project part of state research assignment. We would like to thank Alexey Kashevnik for their feedback and expertise.

\section{REFERENCES}

[1] D. Gavalas, C. Konstantopoulos, K. Mastakas, and G. Pantziou, "Mobile recommender systems in tourism," Journal of Network and Computer Applications, vol. 39, no. 0, pp. 319 - 333, 2014.

[2] S. Balandin and H. Waris, "Key properties in the development of smart spaces," in Proc. 5th Int'l Conf. Universal Access in Human-Computer Interaction (UAHCI '09). Part II: Intelligent and Ubiquitous Interaction Environments, LNCS 5615, C. Stephanidis, Ed. Springer-Verlag, 2009, pp. $3-12$.

[3] J. Honkola, H. Laine, R. Brown, and O. Tyrkkö, "Smart-M3 information sharing platform," in Proc. IEEE Symp. Computers and Communications (ISCC'10). IEEE Computer Society, Jun. 2010, pp. 1041-1046.

[4] K. Kulakov and A. Shabaev, "An approach for creation smart spacebased trip planning service," in Proc. 16th Conf. of Open Innovations Association FRUCT, Oct. 2014, pp. 38-44.

[5] A. Varfolomeyev, D. Korzun, A. Ivanovs, and O. Petrina, "Smart personal assistant for historical tourism," in Recent Advances in Environmental Sciences and Financial Development. Proc. 2nd Int'l Conf. on Environment, Energy, Ecosystems and Development (EEEAD 2014), C. Arapatsakos, M. Razeghi, and V. Gekas, Eds., Nov. 2014, pp. 9-15.

[6] A.-D. N. Tuan-Dung Cao, Thanh-Hien Phan, "An ontology based approach to data representation and information search in smart tourist guide system," Knowledge and Systems Engineering (KSE), 2011 Third International Conference on, pp. 171-175, 2011.

[7] K. Alonso, M. Zorrilla, R. Confalonieri, and J. Vazquez-Salceda, "Ontology-based tourism for all recommender and information retrieval system for interactive community displays," in Information Science and Digital Content Technology (ICIDT), 2012 8th International Conference on. IEEE, 2012, pp. 650-655.

[8] L. Castilloa, E. Armengolb, E. Onaindiac, L. Sebastiac, J. GonzalezBoticariod, A. Rodriguezd, S. Fernandeze, J. Ariase, and D. Borrajoe, "Samap: An user-oriented adaptive system for planning tourist visits," Expert Systems with Applications, vol. 34, pp. 1318-1332, 2008.

[9] A. Morenoa, A. Vallsa, D. Iserna, L. Marina, and J. Borrasb, "Sigtur/edestination: Ontology-based personalized recommendation of tourism and leisure activities," Engineering Applications of Artificial Intelligence, vol. 26, pp. 633-651, 2013.

[10] R. Barta, C. Feilmayr, B. Proell, C. Gruen, and H. Werthner, "Covering the semantic space of tourism - an approach based on modularized ontologies," CIAO '09 Proceedings of the 1st Workshop on Context, Information and Ontologies, no. 1, 2009.

[11] E. Garcia-Barriocanal and M.-A. Sicilia, "On linking cultural spaces and e-tourism: An ontology-based approach," in The Open Knowlege Society. A Computer Science and Information Systems Manifesto. Springer Berlin Heidelberg, 2008, pp. 694-701. 
[12] M. Kalus, "Semantic networks and historical knowledge management: Introducing new methods of computer-based research," Journal of the Association for History and Computing., vol. 10, no. 3, 2007. [Online]. Available: http://hdl.handle.net/2027/spo.3310410.0010.301

[13] V. Mirzaee, L. Iverson, and B. Hamidzadeh, "Computational representation of semantics in historical documents," in Proc. of the XVI International Conf. of the Association for History and Computing, 2005, pp. 199-206.

[14] M. Pasin and J. Bradley, "Factoid-based prosopography and computer ontologies: towards an integrated approach," in Literary and Linguistics Computing, 2013.

[15] M. Doerr and A. Kritsotaki, "Documenting events in metadata," in The 7th International Symp. on Virtual Reality, Archaeology and Cultural Heritage VAST, 2006.
[16] D. Korzun, I. Galov, A. Kashevnik, and B. S., "Virtual shared workspace for smart spaces and m3-based case study," in Proc. 15th Conf. of Open Innovations Association FRUCT. IEEE, Apr. 2014, pp. 60-68.

[17] A. Kashevnik, A. Smirnov, N. Shilov, N. Teslya, and A. Shabaev, "Mobile application for guiding tourist activities: tourist assistant - tais," in Open Innovations Association (FRUCT16), 2014 16th Conference of. IEEE, Oct. 2014, pp. 95-100.

[18] A. Kashevnik, A. Smirnov, N. Shilov, and O. Gusikhin, "Context-driven on-board information support: Smart space-based architecture," in $U l$ tra Modern Telecommunications and Control Systems and Workshop (ICUMT), 2014 6th International Congress on. IEEE, Oct. 2014, pp. 99-104 